\title{
Evaluation of porcine mesenchymal stem cells for therapeutic use in human liver cancer
}

\author{
ARIANE GROTH $^{1,2,3^{*}}$, SABINE OTTINGER $^{1,2^{*}}$, CHRISTIAN KLEIST $^{4}$, \\ ELISABETH MOHR ${ }^{4}$, MOHAMMAD GOLRIZ ${ }^{2}$, DANIEL SCHULTZE ${ }^{2}$, HELGE BRUNS ${ }^{2}$, \\ ARIANEB MEHRABI ${ }^{2}$, PETER SCHEMMER $^{2}$, MARKUS W. BÜCHLER $^{2}$ and INGRID HERR ${ }^{1,2}$ \\ ${ }^{1}$ Molecular OncoSurgery, University of Heidelberg and German Cancer Research Center; ${ }^{2}$ Department of General \\ Surgery, University of Heidelberg, Heidelberg; ${ }^{3}$ Institute of Biomedical Research, Georg-Speyer-Haus, Frankfurt \\ am Main; ${ }^{4}$ Department of Transplantation Immunology, University of Heidelberg, Heidelberg, Germany
}

Received August 3, 2011; Accepted September 9, 2011

DOI: $10.3892 /$ ijo.2011.1217

\begin{abstract}
Mesenchymal stem cell (MSC) transplantation is suggested for therapy of end-stage liver disease, due to e.g. liver cancer and metastasis. Liver transplantation is the only therapeutic option so far but donor organs are short. Also, the availability of allogeneic human MSCs for liver regeneration is limited. Therefore, we evaluated the suitability of porcine bone marrow MSCs from semi-adult pigs and found that morphology, surface expression pattern and multilineage differentiation are similar to those of human MSCs. Porcine MSCs differentiated to a hepatocyte-like phenotype and expressed porcine mRNA of typical liver proteins. However, hepatocyte-like MSCs failed to express the corresponding proteins and did not produce glycogen and urea as primary porcine hepatocytes do. Porcine MSCs were immunotolerated, since they did not activate resting human PBMCs, and were not attacked by human activated PBMCs. However, porcine MSCs led to enhanced proliferation of human pre-activated PBMCs suggesting that immunotoleration of porcine MSCs in the human system has limitations. Together, the potential of porcine MSCs for xenogenous use in human liver therapy is promising but needs further evaluation prior to clinical use.
\end{abstract}

\section{Introduction}

In liver transplantations the number of organs from brain-dead donors is far from enough. Many patients die before a transplant can be assigned to them and liver transplantation is limited by financial aspects and the necessity of lifelong immune suppression (1). Strategies with non-cellular and hepatocyte-based

Correspondence to: Dr Ingrid Herr, Experimental Surgery/MOC, University of Heidelberg, Im Neuenheimer Feld 365, 69120 Heidelberg, Germany

E-mail: i.herr@dkfz.de

*Contributed equally

Key words: mesenchymal stem cells, liver cancer, liver transplantation technologies for liver regeneration are still far behind expectations. The search for alternative cell sources prompted the development of protocols to generate hepatocytes from mesenchymal stem cells (MSC) (2). MSC are adult stem cells isolated from bone marrow or other mesenchymal tissues and they can be easily expanded ex vivo. The term MSC is commonly applied to the plastic-adherent cell culture preparations, which express CD105, CD73 and CD90, but lack expression of haematopoietic markers. Also, MSC must be capable of differentiation to osteoblasts, adipocytes and chondroblasts in vitro (3).

MSC derived from human, rat or mouse bone marrow, umbilical cord blood or adipose tissue have been shown to differentiate into hepatocyte-like cells in vitro upon stimulation with a combination of hepatocyte growth factor (HGF), fibroblast growth factor (FGF) epidermal growth factor (EGF) and other cytokines (4-8). Such MSC-derived hepatocyte-like cells produced albumin, glycogen, urea, low-density lipoprotein and other liver-specific factors $(4,7,9)$. Despite differentiation to hepatocyte-like cells MSC possess immunosuppressive properties, which may be useful use for treatment of liver disease. Human MSC suppress T cell proliferation in response to alloantigens or mitogens $(10,11)$, inhibit $\mathrm{B}$ cell proliferation and antibody production $(12,13)$, inhibit maturation of dendritic cells $(14,15)$, and are not lysed by freshly isolated allogeneic natural killer (NK) cells (16). In patients, autologous transplantation of bone marrow MSC improved short-term liver failure caused by hepatitis B (17), infusion of allogeneic human MSC inhibited acute graft-versus-host disease (GVDH) (18-20), and xenogeneic transplantation of human MSC or conditioned medium of them has been effectively used to reduce rat mortality and the number of apoptotic hepatocytes in experimental liver injury $(21,22)$. Also, engraftment of human MSC occurred after intrauterine transplantation to sheep, even when the transplant was performed after the fetuses became immunocompetent (23). Therefore, human MSC are regarded as non-immunogenic even across species barriers and immunosuppressive properties of MSC have been demonstrated to be maintained upon differentiation to osteoblasts, chondroblasts and adipocytes (24).

While autologous transfer of MSC constitutes the safest approach in cellular transplantation it is mostly associated 
Table I. Comparison of HHMM-P, which induces differentiation of porcine MSC to a hepatocyte-like phenotype, with other non-effective hepatocyte growth media.

\begin{tabular}{lcccc}
\hline Ingredients & HHMM-P & HHMM-A & HGM & William's E \\
\hline Essentials & + & + & + & + \\
Antibiotics & + & + & - & + \\
Cortisone & + & + & + & - \\
Transferrin & + & + & + & + \\
Albumin & + & + & - & - \\
Galactose & + & + & - & - \\
Inositol & - & - & - & + \\
Gluthatione & - & - & + & + \\
BSA & - & + & - & + \\
FCS & - & + & + & - \\
Insulin & + & + & - & - \\
HGF & + & + & + & - \\
EGF & + & + & + \\
\hline
\end{tabular}

Essentials (vitamins, glucose, amino acids, salts, buffer, micronutrients) were present in all four media.

with the need of MSC isolation from aged patients. However, several studies suggest that the quality of MSC decrease with the age of donors (25-29). The disadvantage of aged MSC may be circumvented by allogeneic transplantation of MSC derived from very young donors. But bone marrow donation is painful and extracorporal expansion of MSC from children is limited for ethical reasons. Therefore, xenogenous transplantation of MSC derived from young animals may be an alternative option. Since pigs are genetically closer to humans than rats and mice we used MSC derived from porcine bone marrow of semi-adult pigs for our studies.

In the present study we isolated MSC from porcine bone marrow and found similar morphology, differentiation potential and surface marker expression as described for human MSC. Likewise, porcine MSC were able to differentiate to hepatocyte-like cells, which partially express mRNA of liver specific factors. However, porcine MSC did not differentiate to functional hepatocytes. Porcine MSC did not activate resting peripheral blood monocytes (PBMC) and were themselves not affected by them. However, hepatocyte-like porcine MSC led to enhanced proliferation of anti-CD3 activated PBMC suggesting induction of immune response by porcine MSC in the human environment.

\section{Materials and methods}

Isolation of porcine MSC from BM and culture. Porcine bone marrow was harvested from the iliac crest of six 8 to 12 week-old, healthy German landrace pigs; $40 \mathrm{ml}$ bone marrow aspirate was collected in a syringe containing 10,000 IU heparin to prevent coagulation. The mononuclear cell fraction was isolated by Biocoll density-gradient centrifugation $\left(\mathrm{d}=1.077 \mathrm{~g} / \mathrm{cm}^{3}\right.$; Biochrom, Berlin, Germany). Cells were plated on fibronectin $(10 \mathrm{ng} / \mathrm{ml}$, Sigma, Taufkirchen, Germany)-coated cell culture flasks and selected by plastic adherence upon culture in MSC-NH expan- sion medium (Miltenyi Biotec, Bergisch Gladbach, Germany). All cell culture plastic was purchased from TPP (Trasadingen, Switzerland). Animal experiments have been carried out at the Animal Facility of the University of Heidelberg after approval by the authorities (Regierungspräsidium Karlsruhe, Germany).

MSC marker expression measured by flow cytometry. MSC marker expression was analyzed by FACS measurement using FITC-conjugated antibodies directed toward porcine CD44, CD29, CD105, CD13, CD14, CD45RB and HLA-DR/DP (all antibodies were from Biozol, Eching, Germany). Briefly, $0.5 \times 10^{6}$ cells were incubated with Gammunex ${ }^{\circledR}$ (Talecris Biotherapeutics, Frankfurt, Germany) at $4^{\circ} \mathrm{C}$ for $10 \mathrm{~min}$ to inhibit unspecific binding of antibodies. After washing with $\mathrm{PBS} / 5 \%$ FCS, the cells were incubated with primary antibody and incubated at $4^{\circ} \mathrm{C}$ for $30 \mathrm{~min}$. After washing, cells were resuspended in PBS/5\% FCS and analyzed by flow cytometry. The data were analyzed using BD FACS Diva software (BectonDickinson, Heidelberg, Germany) and compared to isotype controls. Dead cells were excluded by 7-Aminoactinomycin D (7AAD) staining.

Adipogenic and osteogenic differentiation of porcine MSC. For osteogenic and adipogenic differentiation MSC were seeded at a density of $4.5 \times 10^{4}$ cells/well in 6 -well cell culture plates in ready-to-use NH OsteoDiff medium (Miltenyi Biotec Bergisch Gladbach) and ten days later alkaline phosphatase, expressed by osteoblasts was visualized by FAST BCIP/NBT (Sigma), which converts into a deep blue color. For adipogenic differentiation MSC were seeded at a density of $7.5 \times 10^{4}$ cells/ well in 6-well cell culture plates in ready-to-use NH AdipoDiff medium (Miltenyi Biotec Bergisch Gladbach). After 21 days cells were incubated with Oil Red O (Sigma) for $20 \mathrm{~min}$. This lipophilic dye stains the neutral lipid droplets and lipid vacuoles of adipocytes. 
Table II. Sequences established for LightCycler-PCR analysis of porcine liver-specific factors.

\begin{tabular}{ll}
\hline Name & \multicolumn{1}{c}{ Primer sequences } \\
\hline TFN-for & 5'-TGC ACC ATT TCA AAT CAG GA-3' \\
TFN-rev & 5'-GAG AAG AAA CTG GCC ACT GC-3' \\
ALB-for & 5'-ACG AAT GCT TCC TCC AAC AC-3' \\
ALB-rev & 5'-TCT GCA CAT TCA AGC AGG TC-3' \\
AFP-for & 5'-TCG CAG TCT GAA AAC CCT CT-3' \\
AFP-rev & 5'-TTG CAG TGC TAC ACC CTG AG-3' \\
LPL-for & 5'-TGG ACG GTG ACA GGA ATG TA-3' \\
LPL-rev & 5'-AGG TGA CCC TCT GGT GAA TG-3' \\
Cx43-for & 5'-GTC GGT CGT ATC CTT GGT GT-3' \\
Cx43-rev & 5'-CAG TTT TGC TCA CTC GCT TG-3' \\
Cyp7A1-for & 5'-GAA GCA TTG ACC CCA GTG AT-3' \\
Cyp7A1-rev & 5'-ACC AGT TCC GAG ATG TGG TC-3' \\
TTR-for & 5'-ATG GTC AAA GTC CTG GAT GC-3' \\
TTR-rev & 5'-TTG TCT CTG CCC GAG TTT CT-3' \\
CK19-for & 5'-CGA ACC AAG TTT GAG ACG GAA C-3' \\
CK19-rev & 5'-CCG CTG GTA CTC CTG ATT CTG C-3' \\
PCK1-for & 5'-TGG AGG AAG AGG GTG TCA TC-3' \\
PCK1-rev & 5'-GAG CGT CAG CTC TGG GTT AC-3' \\
18S_for & 5'-AAC CAG ACA AAT CGC TCC AC-3' \\
18S_rev & 5'-GAC CAT AAA CGA TGC CGA CT-3' \\
\hline
\end{tabular}

Isolation of primary hepatocytes from porcine liver. Porcine hepatocytes were isolated according to a two-step collagenase perfusion procedure described for isolation of human hepatocytes $(30,31)$. After perfusion, the liver capsule was incised, the thick fibrous connective tissue was discarded and cell suspensions were harvested. The cells were filtered and purified over a $36 \%$ Percoll gradient (1.130 g/ml Amersham Biosciences). Cells were resuspended in HHMM medium derived from Primacyt (Cell Culture Technology GmbH, Schwerin, Germany) supplemented with $200 \mu \mathrm{g} / \mathrm{ml}$ streptomycin, $200 \mathrm{U} / \mathrm{ml}$ penicillin and $2.5 \mu \mathrm{g} / \mathrm{ml}$ amphotericin B and seeded on collagen I coated cell culture plates. Viability of porcine hepatocytes was greater than $90 \%$ as determined by trypan blue exclusion.
Hepatocytic differentiation of porcine MSC. For induction of hepatocytic differentiation four different media and culture conditions were tested. MSC were seeded to full confluence in each medium and hepatocytic differentiation was evaluated one, two, three and four weeks later. The media used were i) human hepatocyte growth medium as described by Aurich et al for hepatic differentiation of human bone marrow-derived MSC (7). We refer to this medium as HHMM-A in the following; ii) ready-to-use hepatocyte growth medium (HGM) for longterm cultivation of human hepatocytes (PromoCell Heidelberg, Germany), iii) Williams E medium (Sigma) as described (32), or iv) serum-free human hepatocyte maintenance medium obtained from Primacyt (Cell Culture Technology $\mathrm{GmbH}$, Schwerin, Germany) - we refer to this medium as HHMM-P in the following. The composition of the media is summarized in Table I.

RNA isolation and Light Cycler RT-PCR. Total cellular RNA from $5 \times 10^{5} \mathrm{MSC}$ was prepared with the Classic Line E.Z.N.A. ${ }^{\circledR}$ Total RNA kit (PeqLab, Erlangen, Germany) according to the protocols recommended by the manufacturer. RNA concentration and purity were tested by absorption at 260 and $280 \mathrm{~nm}$. For quantitative LightCycler ${ }^{\circledR}$-PCR $1 \mu \mathrm{g}$ RNA was converted to cDNA with the First Strand cDNA Synthesis kit for RT-PCR (Roche Diagnostics GmbH, Mannheim, Germany). cDNA was diluted 1:5 and used for the LightCycler ${ }^{\circledR}-$ PCR. Porcine primer sequences were designed by modification of known functional human primers (Table II). Optimal PCR conditions were established for each primer sequence (Table III). LightCycler FastStart DNA Master SYBR Green I (Roche Diagnostics $\mathrm{GmbH}$, Mannheim, Germany) was used for hot-start PCR in $20 \mu \mathrm{l}$ glass capillaries. Relative quantitative gene expression was calculated by normalization of the data to $18 \mathrm{~S}$ RNA.

Protein isolation and Western blot analysis. Cell proteins were isolated and Western blot analysis was performed as described recently (33). Antibodies reactive with porcine proteins were sheep polygonal Ab anti-Prealbumin, mouse $\mathrm{mAb}$ antiLipoprotein lipase (Abcam, Cambridge, UK) and mouse mAb anti-Albumin (AbD Serotec, Düsseldorf, Germany).

Evaluation of glycogen and urea production. Glycogen deposits were visualized in cells fixed in acetone/methanol by conven-

Table III. Conditions used in LightCycler-PCR analysis of liver specific factors in porcine.

\begin{tabular}{lcccc}
\hline Name & RT reaction time min & Annealing temperature ${ }^{\circ} \mathrm{C}$ & $\mathrm{MgCl}_{2}$ & $\mathrm{No.}_{\text {of cycles }}$ \\
\hline TFN & 60 & 63 & $3 \mathrm{mM}$ & - \\
ALB & 60 & 63 & - & 40 \\
AFP & 60 & 63 & - & - \\
LPL & 60 & 63 & - & 40 \\
Cx43 & 60 & 63 & $3 \mathrm{mM}$ & 40 \\
Cyp7A1 & 60 & 63 & $3 \mathrm{mM}$ & 40 \\
TTR & 60 & 63 & - & 40 \\
CK19 & 60 & 63 & 67 & 40 \\
PCK1 & 60 & 63 & 40
\end{tabular}


tional periodic acid-Schiff (PAS) staining - PAS was from Sigma. Urea concentration in the cell culture supernatant was determined by the colorimetric assay according to Zawada et al (34).

Xenogeneic MSC-lymphocyte reaction and kill assay. Porcine $\operatorname{MSC}\left(5 \times 10^{2}\right)$ in a volume of $100 \mu \mathrm{l} \mathrm{NH}$-expansion medium were seeded in regular 96-well plates or in the lower well of 96-well transwell plates. Human peripheral blood monocytes (PBMC) obtained from healthy donors by Ficoll density centrifugation $\left(1 \times 10^{3}\right)$ were added directly to the MSC or to the upper well of the transwell plate. PBMC were either resting or activated with anti-CD3 antibody for $24 \mathrm{~h}$. Resting or activated PBMC and MSC alone served as controls. Ninty-six hours after incubation $1 \mathrm{Ci} /$ well of tritiated $\left[{ }^{3} \mathrm{H}\right]$-thymidine (Amersham Bioscience) was added. After overnight incubation cells were harvested (Inotec Biosytems, Rockville, MS, USA) onto glass fibre filter mats (Inotec Biosytems, Rockville, MS, USA). Filter mats were allowed to dry at $56^{\circ} \mathrm{C}$ for $30 \mathrm{~min}$ and sealed in sample bags containing $4 \mathrm{ml}$ scintillation fluid (Betaplate Scint). Filter mats were analyzed on a Microbeta $1450^{\mathrm{TM}}$ liquid scintillation counter to determine ${ }^{3} \mathrm{H}$-thymidine uptake by cells. For kill assays, $1.25 \times 10^{5} \mathrm{MSC}$ were seeded in 6-well plates in a volume of $3 \mathrm{ml} \mathrm{NH}$-expansion medium. After adherence, IL-2 and antiCD3-activated PBMC were added in a ratio of 1:1 or 1:5 and co-incubated for $6 \mathrm{~h}$. Apoptosis induction in MSC was analyzed by PI and AnnexinV-PE staining followed by flow cytometry.

Measurement of apoptosis. Cells were stained with fluoresceinisothiocyanate (FITC)-conjugated annexin V (Invitrogen, Camarillo, CA, USA). Externalization of phosphatidylserine was identified by flow cytometry (FACScan, BD Biosciences, Heidelberg, Germany) (33). Likewise, cells were stained with Nicoletti buffer as described (35) and DNA fragmentation was identified by flow cytometry (FACScan, BD Biosciences).

Statistical analysis. Data are presented as the means $\pm \mathrm{SD}$.

\section{Results}

MSC from porcine bone marrow show typical features of human MSC. Bone marrow $(40 \mathrm{ml})$ was harvested from the iliac crest of young ( 8 to 12 week-old) healthy German landrace pigs immediately on sacrifice. It should be noted that these animals did not die for donation of bone marrow only but served before as a model for evaluation of surgical techniques in our department. Mononuclear cells were isolated by Biocoll densitygradient centrifugation, culture in NH-MSC expansion medium and selection of plastic adherent cells. We obtained in average about $1 \times 10^{6} \mathrm{MSC}$ in passage 0 and $2.5 \times 10^{6} \mathrm{MSC}$ in passage 1 . In general, MSC were passaged upon $80 \%$ confluence and medium was renewed twice per week according to culture conditions suggested for human MSC (36). Porcine MSC were growing fast and could be expanded up to 20 passages before reaching senescence. Porcine MSC had a typical spindle-shaped morphology (Fig. 1A), consistent with the morphology of human MSC (37). Corresponding to human MSC, the surface expression pattern of porcine MSC was negative for the hematopoietic antigens CD13, CD14, CD45RB, HLA-DR/DP and positive for the MSC marker pattern CD44, CD29 and CD105 as determined by

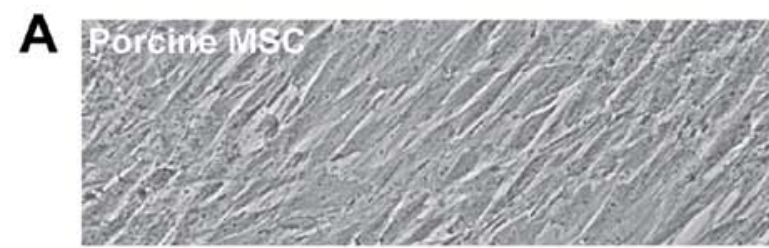

B
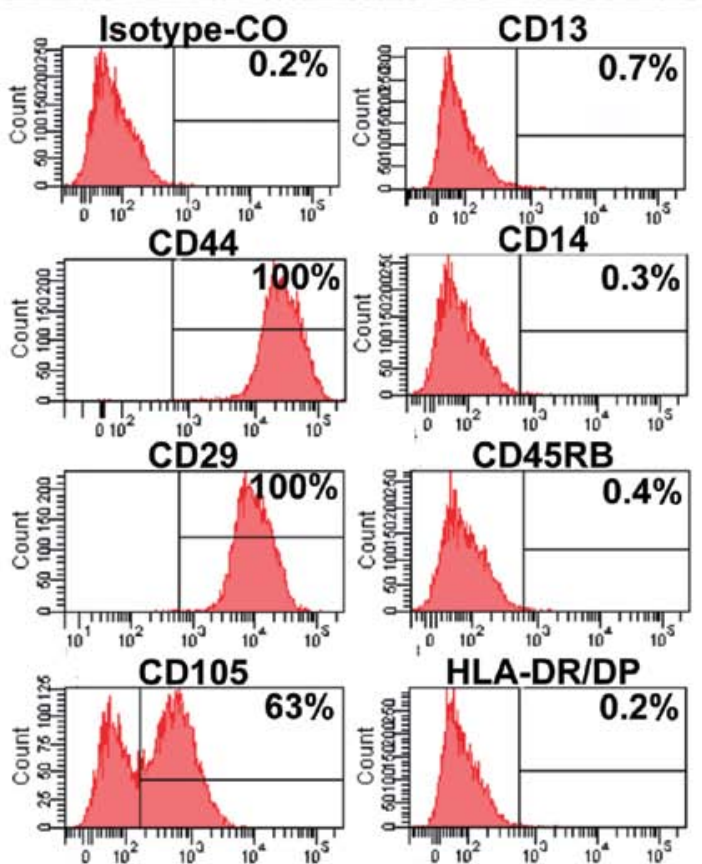

HLA-DR/DP
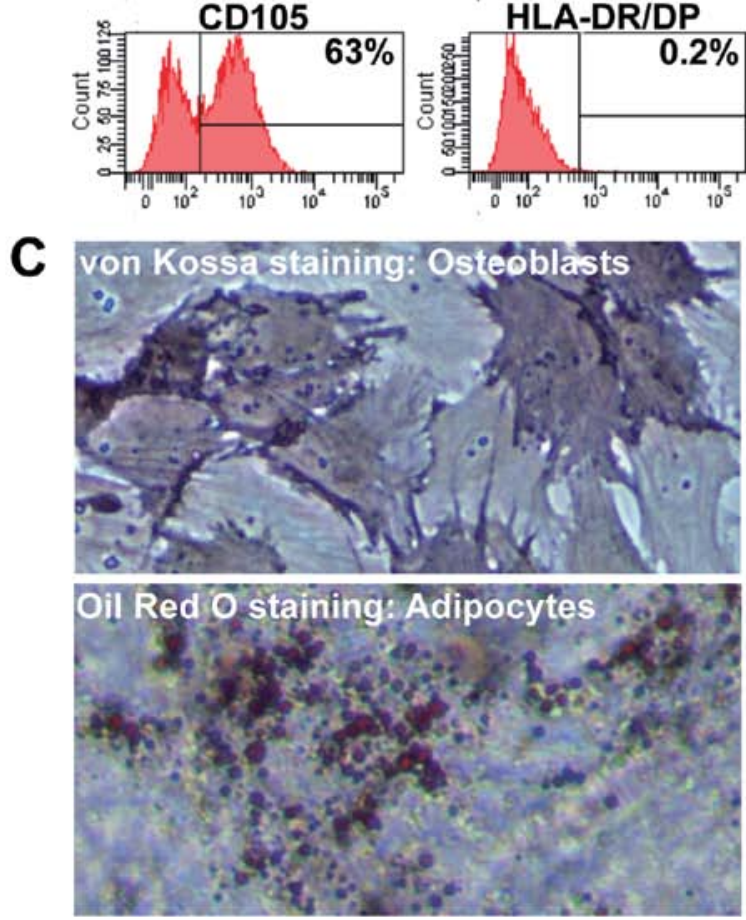

Figure 1. Porcine bone marrow MSC resemble the human counterparts (A) MSC isolated from porcine bone marrow show a spindle shaped phenotype. (B) Typical surface expression markers were evaluated by FACS-analysis of CD44, CD29, CD105, CD13, CD14, CD45RB and HLA-DR/DP, as indicated. Secondary antibodies were FITC- or PE-labeled and isotype antibodies served as control. One representative analysis out of three independent experiments with similar outcome is shown. (C) Differentiation potential of porcine MSC in passage two after incubation in osteogenic or adipogenic differentiation medium for ten or 21 days, respectively. Alkaline phosphatase expressed by osteoblasts was detected by BCIP/NBT and lipid droplets of adipocytes by Oil Red O. Cells were evaluated under 10x magnification using a Nikon Eclipse TS100 microscope. Data are representative of three independent experiments with similar results.

staining with specific antibodies and FACS analysis (Fig. 1B). Equivalent to human MSC, porcine MSC exhibited osteoblastic 

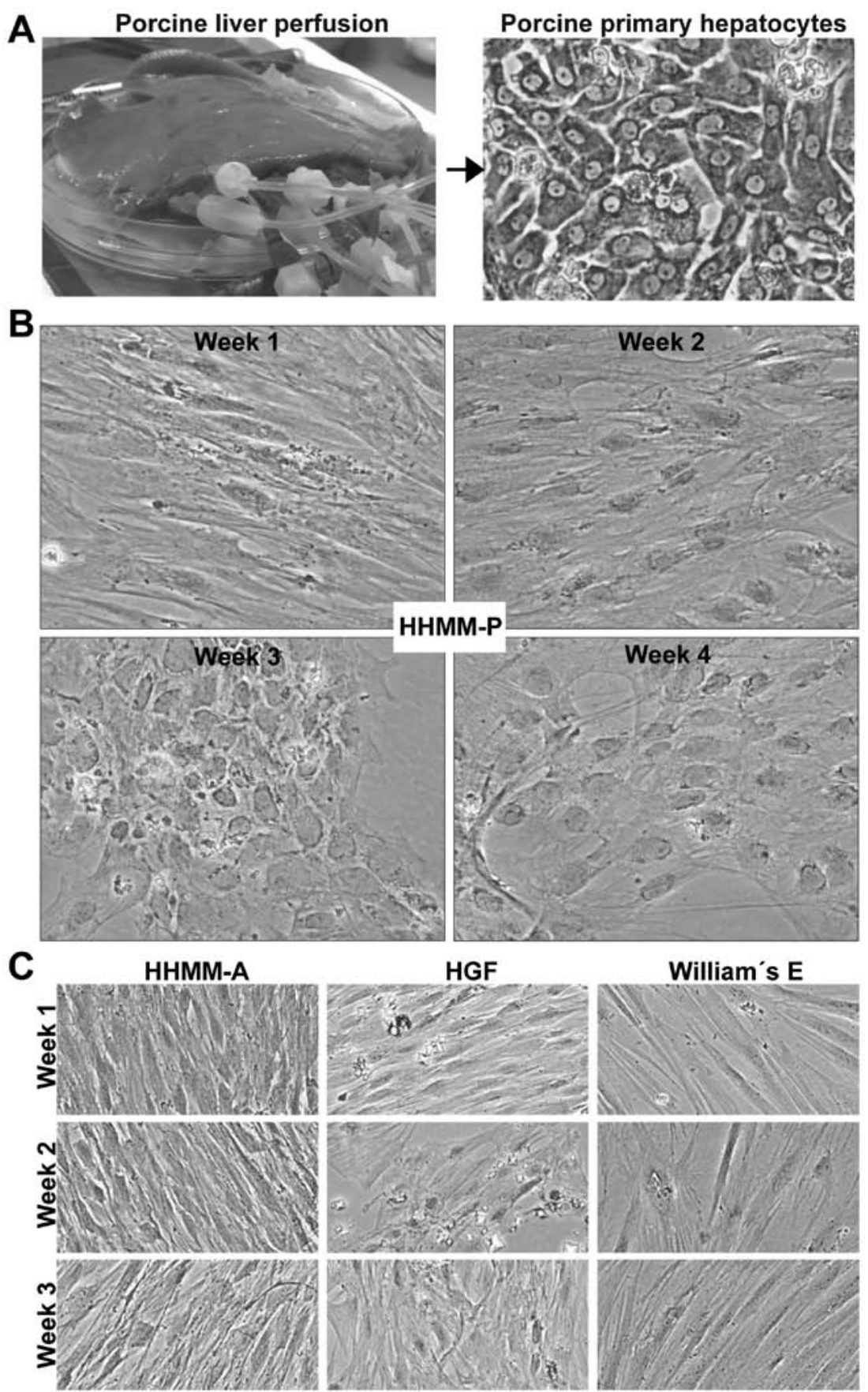

Figure 2. Differentiation of porcine MSC to a hepatocyte-like phenotype. (A) Primary hepatocytes were isolated from porcine liver by a two-step collagenase perfusion procedure and seeded in HHMM-P medium (Primacyt) at a density of $1.5 \times 10^{6} / \mathrm{ml}$. The typical polygonal morphology of hepatocytes was documented under 100x magnification. (B) Porcine MSC were divided in four aliquots at passage 2. Aliquot 1 was cultured in HHMM-P, (C) aliquot 2 in HHMM-A, aliquot 3 in ready-to-use hepatocyte growth medium HGF and aliquot 4 in William's E hepatocyte maintenance medium. Hepatocytic differentiation was phenotypically evaluated one, two, three and four weeks after incubation. Cellular morphology was documented by 100x magnification. Data are representative of three independent experiments with similar results.

and adipocytic differentiation potential as detected by culture of MSC in specific differentiation media followed by visualization of bone structures by von Kossa staining and of fat droplets by Oli-red O staining (Fig. 1C).

Differentiation of porcine MSC to a hepatocyte-like phenotype. For evaluation of the hepatic differentiation potential we cultured aliquots of MSC derived from the same pig in four growth and maintenance media commonly used for culture of human hepatocytes. HHMM-P obtained from Primazyt is a serum-free human hepatocyte maintenance medium as described (38); HHMM-A is a modified FCS-containing human hepatocyte maintenance medium as described for hepatocytic differentiation of human MSC (7); HGM is a hepatocyte growth medium optimized for culture of human hepatocytes, which has been evaluated for culture of porcine hepatocytes 


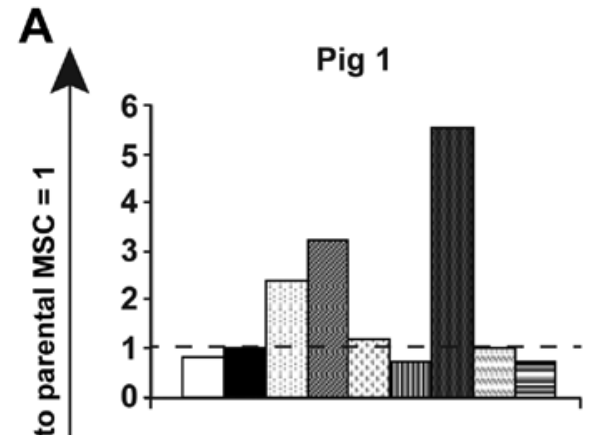

Pig 2

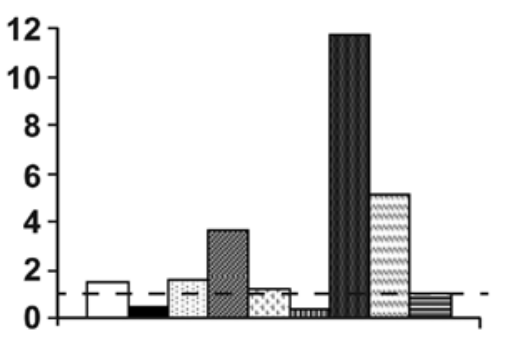

Pig 3

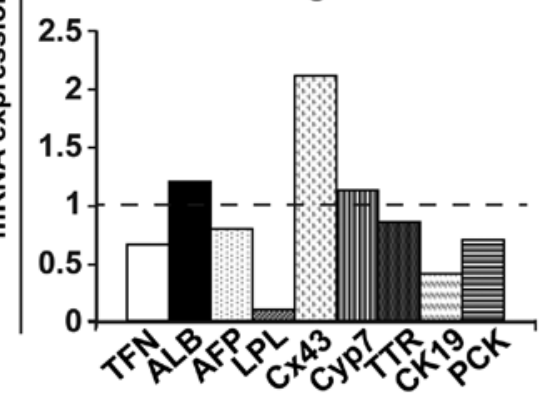

Pig 4

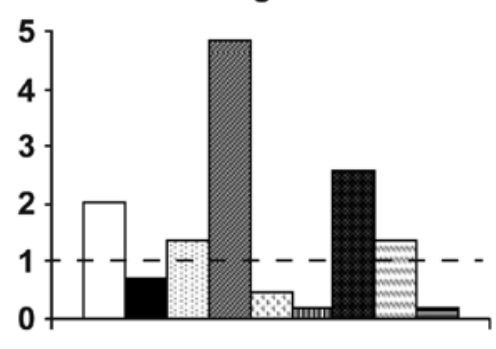

Pig 5

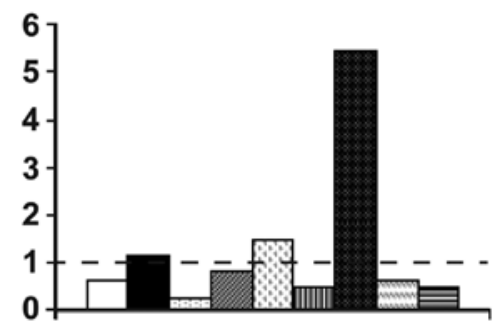

Pig 6

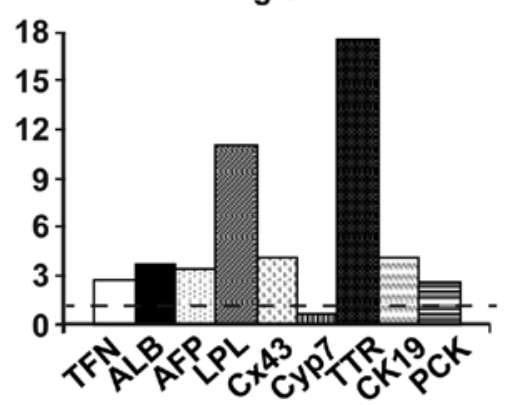

B

\begin{tabular}{|c||c|c|c|c|c|c|c|c|c|}
\hline & TFN & ALB & AFP & LPL & Cx43 & Cyp7 & TTR & CK19 & PCK \\
\hline Pig 1 & - & - & + & + & + & - & + & - & - \\
\hline Pig 2 & + & - & + & + & + & - & + & + & - \\
\hline Pig 3 & - & + & - & - & + & + & - & - & - \\
\hline Pig 4 & + & - & + & + & - & - & + & + & - \\
\hline Pig 5 & - & + & - & - & + & - & + & - & - \\
\hline Pig 6 & + & + & + & + & + & - & + & + & + \\
\hline Total + & 3 & 3 & 4 & 4 & 5 & 1 & 5 & 3 & 1 \\
\hline
\end{tabular}

Overexpression of hepatocyte-specific mRNA by pig hepatocyte-like MSC

Figure 3. Hepatocyte-like MSC overexpress liver-specific mRNA. (A) Total RNA was extracted from parental and hepatocyte-like porcine MSC three-weeks after culture in HHMM-P medium. MSC were derived from 6 different pigs. RNA transcripts typically expressed in hepatocytes, such as the early- (AFP, CK19, Cx43) or late-hepatocyte markers (ALB, PCK1, TFN) and other liver cell-specific factors (LPL, Cyp7, TTR) were detected by light Cycler RT-PCR. Signals were normalized to the amount of 28S RNA transcripts, which served as internal standard. The expression pattern is calculated as fold induction of hepatocyte-like MSC relative to non-differentiated parental MSC, which are set to 1. (B) Table in which the results of the RT-PCR are summarized. Overexpression (+), nonoverexpression (-).

(PromoCell); William's E (Sigma) is a classical FCS-containing medium described for enrichment of isolated hepatocytes and reducing the number of contaminating fibroblasts (32). To have a master copy of the appearance of MSC after hepatic differentiation, we isolated hepatocytes from porcine liver by collagenase perfusion. Porcine hepatocytes showed typical polygonal morphology of human hepatocytes (Fig. 2A). A similar phenotype was acquired by porcine MSC within two weeks of incubation in HHMM-P medium: MSC converted their spindle shaped form to the polygonal phenotype of hepatocytes (Fig. 2B). The phenotypical changes increased with prolonged culture of MSC in HHMM-P medium for three and four weeks. In contrast, incubation of MSC in HHMM-A, HGF or William's E medium did not induce phenotypical changes (Fig. 2C). We also tried to differentiate porcine MSC to hepatocyte-like cells according to a protocol described for hepatic differentiation of human bone marrow-derived MSC (7). During this procedure human MSC are pretreated with 


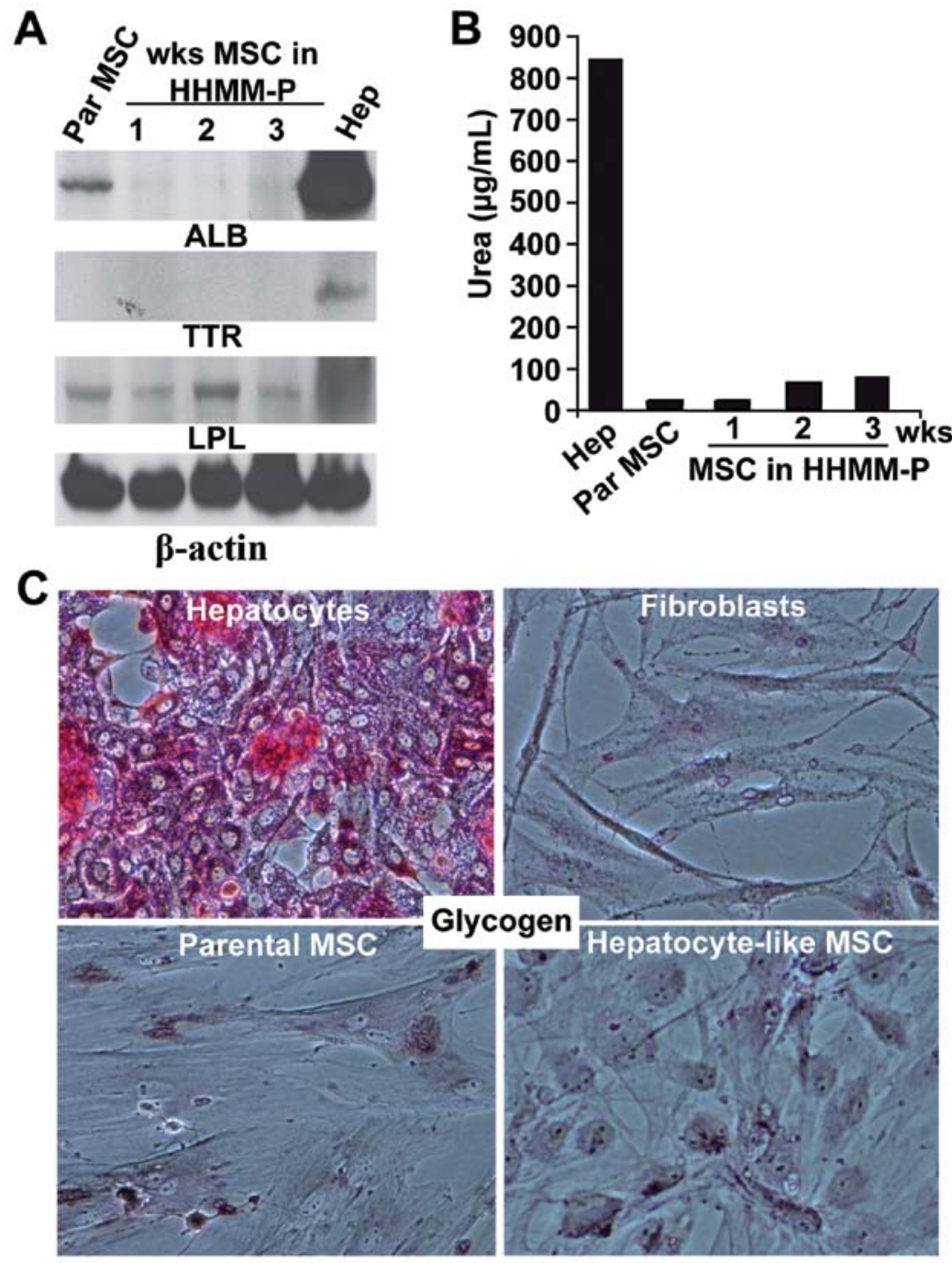

Figure 4. Hepatocyte-like MSC do not overexpress liver-specific proteins, urea and glycogen. (A) Protein was extracted from porcine hepatocytes (Hep), and MSC derived from pig 6, before (Par MSC) and 1,2 and three weeks after cultivation in HHMM-P as indicated. Western blot analysis was performed using specific antibodies for detection of porcine albumin (ALB), transthyretin (TTR) and lipoprotein lipase (LPL). $\beta$-actin served as control for equal conditions. (B) For analysis of urea synthesis supernatants from cells growing in equal density were collected and analyzed as described in the Materials and methods section. (C) Glycogen was detected by PAS staining in primary porcine hepatocytes, human skin fibroblasts, parental porcine MSC or Hepatocyte-like MSC after incubation in HHMM-P for 3 weeks. Data are representative of three independent experiments with similar results.

$20 \mu \mathrm{M} 5$-azacytidine for $24 \mathrm{~h}$, followed by replacement of the growth medium by HHMM-A. However, 5'-azacytidine treatment was toxic for porcine MSC and induced rapid cell death (data not shown). To identify factors responsible for hepatocyte-like differentiation of porcine MSC we compared ingredients of HHMM-P medium, which induces differentiation of porcine MSC to a hepatocyte-like phenotype, with the three other media, which did not (Table I). All media contained essential ingredients of a cell culture media, namely vitamins, glucose, amino acids, salts, buffer and micronutrients, although in different composition and concentration. Similar to HHMM-A, the successful HHMM-P medium contained HGF and EGF, while in HGM only EGF and in William's E none of these growth factors were present. The only obvious difference between HHMM-P and HHMM-A is the presence of FCS in HHMM-A but not in HHMM-P. Thus, HGF and EGF are necessary for hepatocyte-like differentiation of porcine MSC, while FCS may counteract hepatic differentiation of porcine MSC despite the presence of HGF and EGF.

Porcine hepatocyte-like MSC express liver-specific mRNA. For analyzes of mRNA expression before and three weeks after HHMM-P induced hepatocyte-like differentiation of porcine MSC, we performed light-cycler RT-PCR. RNA harvested from six different pigs was evaluated. Since porcine genes are rarely published and primer oligonucleotides are not available commercially, we designed primer sequences according to pig gene sequences already published and established the appropriate light cycler conditions (Tables I and II). mRNA overexpression of porcine hepatocyte-like MSC relative to parental MSC is shown (Fig. 3A). While in each pig overexpression of some but not all of nine examined genes was visible, the highest rate of overexpression was found in pig 6 (Fig. 3B). Among overexpressed genes are 
early hepatogenic differentiation markers $\alpha$-fetoprotein (AFP) and cytokeratin-19 (CK19). Moreover, transcripts for markers of well-differentiated hepatocytes such as the xenobiotic metabolism enzyme cytochrome P450 subtype 7 (CYP7), the cytosolic key control enzyme of gluconeogenesis, phosphoenolpyruvate carboxykinase (PCK1), the hepatic gap junction protein connexin $32(\mathrm{Cx} 32)$ and the secretory plasma proteins albumin (ALB) and transferrin (TFN) were upregulated. In addition, prealbumin, also known as trans-thyretin (TTR) and lipoprotein lipase (LPL), a liver protein of the perinatal period, were upregulated.

Porcine hepatocyte-like MSC lack expression of liver-specific proteins. By using proteins isolated from parental and hepatocyte-like differentiated MSC from pig 6 we examined expression of liver-specific proteins albumin, transthyretin (prealbumin) and lipoprotein lipase. Unexpectedly, no upregulation of these proteins could be detected compared to porcine hepatocytes and parental MSC (Fig. 4A). We could not examine more liver-specific proteins, since availability of pig-specific antibodies is limited. We also examined other hepatocyte specific-features such as urea synthesis and glycogen storage. However, 1, 2 or 4 weeks after initiation of hepatocyte-specific differentiation porcine MSC did not overexpress urea (Fig. 4B) or glycogen (Fig. 4C). No significant difference to parental MSC or fibroblasts could be detected. In contrast, urea and glyocogen production was highly upregulated in primary porcine hepatocytes. These data suggest that porcine MSC are able to differentiate to a hepatocyte-like phenotype, which, however, is not able to fully overtake hepatic function.

Porcine MSC are partially immunotolerated by human PBMC. Another function of human MSC important for liver transplantation and regeneration is their proposed immunosuppressive potential $(20,39)$ along with immuno acceptance across species barriers (21-23). Considering a potential use of porcine MSC in therapy of patients we evaluated whether porcine MSC may evoke immune responses and may be tolerated by human immune cells or not. PBMC, isolated from blood of human healthy donors, were co-incubated with parental porcine MSC. Co-incubations were performed in transwell plates to examine the effect of released factors or in direct co-incubation assays to examine effects of cell-cell contact and soluble factors. Ninty-six hours later we measured the activation status of PBMC by ${ }^{3} \mathrm{H}$-thymidine incorporation and scintillation. Compared to CD3-activated PBMC, which served as positive control, no activation of resting PBMC upon co-incubation with MSC occurred (Fig. 5A). To examine whether MSC are able to reduce proliferation of activated PBMC we pre-treated PBMC with CD3 followed by co-incubation with MSC. However, this did not result in reduced proliferation of MSC as expected, but increased activity of PBMC in both co-culture assays and induction was even stronger in the direct co-culture assay (Fig. 5A). To test whether porcine MSC are tolerated by human activated PBMC we directly co-incubated the cells. Fourty-eight hours later we measured apoptosis induction in MSC by annexin and propidium iodide staining followed by FACS analysis (Fig. 5B). Basal apoptosis rate of MSC was not significantly enhanced by co-culture with PBMC at a ratio of 1:1 or 1:5. Collectively, porcine MSC do not affect resting PBMC but enhance prolif-

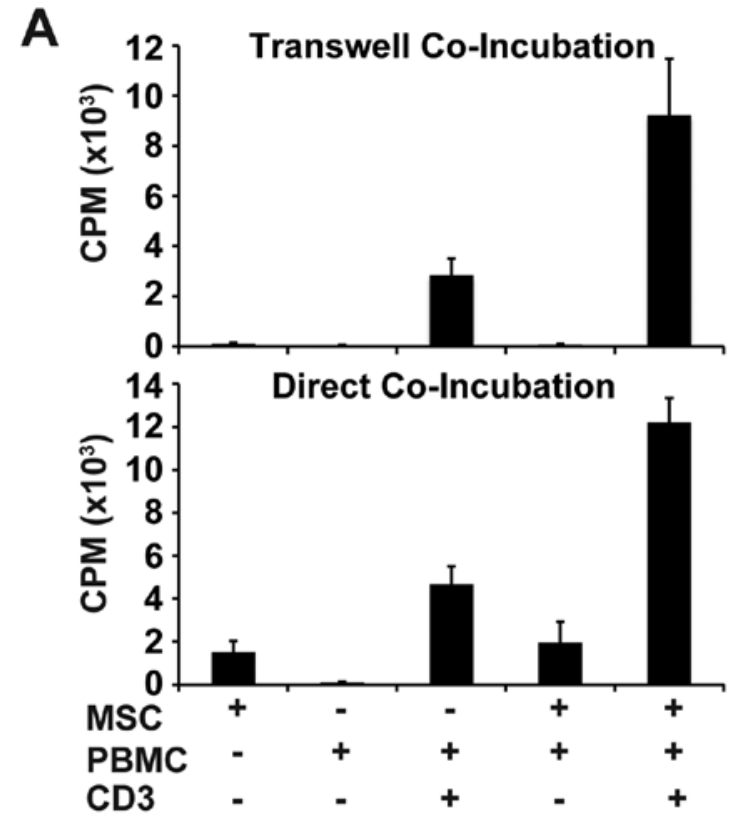

B

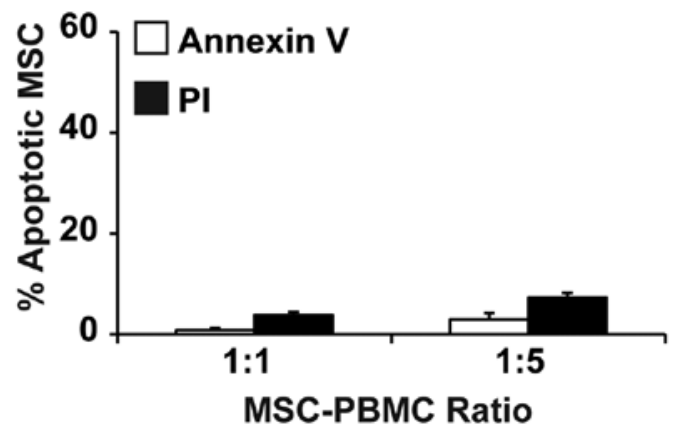

Figure 5. Porcine MSC are tolerated by human PBMC but superinduce them (A) Allogen reaction. Human primary PBML $\left(1 \times 10^{3}\right)$ and parental porcine MSC $\left(5 \times 10^{2}\right)$ were incubated alone or mixed together (ratio of PBMC:MSC $=1: 0.5$ ). As a positive control, PBMC were stimulated with CD3 (50 ng/ml) for $24 \mathrm{~h}$. MSC were either directly co-incubated with PBMC (Direct Co-Incubation) or in Transwell Plates (Transwell Co-Incubation) for $96 \mathrm{~h}$. Proliferation of PBMC was measured by ${ }^{3} \mathrm{H}$-thymidine incorporation and scintillation. (B) Kill assay. A total of $1.25 \times 10^{5}(1: 1)$ or $6.25 \times 10^{5}$ (1:5) CD3-activated human PBMC was added to $1.25 \times 10^{5}$ adherent growing parental porcine MSC. Forty-eight hours later, PBMC were removed by washing and apoptosis in MSC was measured by staining of cells with annexin $\mathrm{V}$ and propidium iodide, followed by FACSanalysis. Data are representative of three independent experiments with similar results and $\mathrm{SD}$ are shown.

eration of CD3 pre-activated PBMC, while activated PBMC do not attack MSC.

\section{Discussion}

In the present study we demonstrate that porcine MSC share essential features with human MSC such as morphology, marker expression and multi-lineage differentiation potential e.g. to adipocytes and osteoblasts (3). Moreover, we were able to differentiate porcine MSC to hepatocyte-like cells by culture in serum-free human hepatocyte maintenance medium (HHMM-P) supplemented with human HGF and EGF. Within 
2-3 weeks of culture porcine MSC acquired a hepatocyte-like phenotype similar as described by Stock et al for human MSC (2). However, the media suited for hepatic differentiation seem to differ between porcine and human MSC. Porcine MSC did not differentiate in HHMM-A medium, which is suggested by Aurich and colleagues for hepatic differentiation of human MSC $(2,7,8)$. Instead, porcine MSC preferred HHMM-P medium from Primazyt, which was the only medium suited for hepatocytelike differentiation of porcine MSC out of four different media evaluated. The only difference of HHMM-A and HHMM-P is the presence of fetal calf serum in HHMM-A. Thus, fetal calf serum seems to be an inhibiting factor for porcine but not for human MSC regarding hepatocyte-like differentiation. In addition, our experiments reveal that HFG and EGF are essential for hepatocyte-like differentiation of porcine MSC: these growth factors are present in HHMM-P, while HGM medium, which does not induce hepatocyte-like differentiation of porcine MSC, contains EGF only and lacks HGF and fetal calf serum. Likewise, William's E medium contains fetal calf serum but no human growth factors and did not lead to hepatocyte-like differentiation of porcine MSC. Our results are promising, since the composition of HHMM-P is closest to the environment in human liver: absence of fetal calf serum but presence of human HGF and EGF. This makes it likely that parental porcine MSC transplanted to human liver may acquire a hepatocyte-like phenotype or that the phenotype of porcine hepatocyte-like MSC is maintained after transplantation. There is another difference between the human and porcine differentiation system, since we found that porcine MSC do not tolerate pre-treatment with 5'-azacytidine, a chemical analogue of cytidine used as chemotherapeutic agent. While this chemical is routinely used for initiation of hepatocyte-differentiation of human MSC by demethylation (2,7,8), the porcine counterparts do not survive pre-treatment with 5'-azacytidine in concentrations and incubation times used for human MSC.

We evaluated liver-specific mRNA expression in porcine hepatocyte-like MSC three weeks after initiation of differentiation. We found enhanced mRNA expression in eight of nine examined liver-specific markers in MSC derived from pig 6 with most prominent overexpression of transthyretin and lipoprotein lipase. Transthyretin was also overexpressed in 4 and lipoprotein lipase in 3 other pigs. These and additional liver-specific genes were overexpressed in some but not in all pigs. Overall, the expression patterns and levels of liver-specific mRNAs were not consistent between hepatocyte-like MSC derived from 6 pigs in total. This may be due to individual differences between animals together with different quality and passage numbers of individual MSC lines. In addition, it may well be that the time point of examination three weeks after initiation of differentiation was too late. In line with this assumption, Runge et al found that primary hepatocytes lose their functional abilities in serum-free long-term culture (38). This seems to be a consequence of molecular downregulation rather than cellular de-differentiation, as all functional abilities are restored once the cells are transplanted into a recipient liver $(40,41)$. Differentiated MSC are suggested to lose their functional abilities also in extended cultures, and Stock et al recommend that hepatocyte-like human MSC should not be grown for more than 21 days (2). To consider this recommendation we evaluated protein expression of albumin, transthyretin and lipoprotein lipase in MSC derived from the most promising pig 6 after 1,2, and 3 weeks of hepatocytelike differentiation. However, compared to parental MSC and primary porcine hepatocytes we did not see protein overexpression. Similarly, urea and glycogen were basally expressed by parental MSC but not enhanced in hepatocyte-like MSC.

The aim of the presented study was the analysis of porcine MSC and their suitability for use in xenotransplantation to the human liver. Therefore, we evaluated immunological feature of porcine MSC upon co-incubation with human PBMC. While allogeneic transplanted MSC are suggested to be tolerated and to suppress alloreactivity $(18,20,42)$, the literature has few data on immune responses after xenogeneic MSC transplantation. Most studies with human MSC have used immunologically incompetent animals as summarized in a recent review by Poncelet $e t$ al (43). One in vitro study demonstrates that minipig-derived bone marrow MSC actively inhibit proliferation of human $\mathrm{T}$ cells. This result was promising and is important for our approach (44). Saito et al (45) confirmed immunotoleration of MSC in the xenogenous setting as demonstrated by engraftment of mouse MSC to injured myocardium of immunocompetent rats. The findings of xenogenous immunotoleration could be only partially confirmed by our experiments. While porcine MSC in our system did not lead to proliferation of human resting PBMC, which is consistent with the reports above, porcine MSC increased proliferation of CD3-activated PBMC. Simultaneously we observed partial immunotolerance, since human PBMC did not induce apoptosis in porcine MSC upon co-incubation. Our results reflect data obtained in an intracardiac human-to-rat system, where rat $\mathrm{T}$ cell proliferation was increased after a single dose of human MSC in immunocompetent rats (46). In this study tacrolimus-based immunosuppression abrogated immune sensitization. Without immunosuppression, an intense local macrophage-mediated cellular response occurred after MSC transplantation. Differences in MSC species could explain these contradictory findings e.g. different expression of histocompatibility complex (MHC) class II proteins. When considering the pig as a potential source of xenogeneic cells, attention should be focused on a role for the Gal- $\alpha 1,3-\mathrm{Gal}$ epitope expressed on subgroups of porcine cells and secondly on molecules involved in triggering antibody-independent natural killer (NK) cellmediated cytotoxicity $(43,47,48)$. No studies to date have focused on the expression of the Gal- $\alpha 1,3-G a l$ epitope on MSC, but some studies suggest that bone marrow progenitor cells express no or little Gal- $\alpha 1,3-\mathrm{Gal}$ (49) even though conflicting data were reported on porcine embryonic stem cells (50).

In conclusion, our data indicate that porcine MSC closely resemble human MSC, grow in human MSC medium and acquire a hepatocyte-like phenotype induced by human EGF and HGF. Although hepatocyte-like differentiated porcine MSC do not overtake hepatic function, they may be suited for reconstituting the parenchyma of damaged liver. However, the use of porcine MSC in human liver therapy may require immunosuppression, since although porcine MSC did not activate resting PBMC nor were killed by activated PBMC, further activation of CD3-preactivated human PBMC by porcine MSC occurred. While the obtained data are promising further research is needed to understand interaction of porcine MSC with liver function and immunoregulatory cells in the human system. 


\section{Acknowledgements}

We thank Dr A. Salnikov for helpful discussion. This study was supported by grants from the Frontier Excellence Initiative of the University of Heidelberg, Dietmar-Hopp Stiftung, Deutsche Krebshilfe (109362) and DFG (HE 3186/11-1). All authors disclose there is no commercial association that might create a conflict of interest in connection with the submitted manuscript. There are no competing financial interests.

\section{References}

1. Demetriou AA, Brown RS Jr, Busuttil RW, Fair J, McGuire BM, Rosenthal P, Am Esch JS II, Lerut J, Nyberg SL, Salizzoni M, Fagan EA, de Hemptinne B, Broelsch CE, Muraca M, Salmeron JM, Rabkin JM, Metselaar HJ, Pratt D, De La Mata M, McChesney LP, Everson GT, Lavin PT, Stevens AC, Pitkin Z and Solomon BA: Prospective, randomized, multicenter, controlled trial of a bioartificial liver in treating acute liver failure. Ann Surg 239: 660-770, 2004.

2. Stock P, Bruckner S, Ebensing S, Hempel M, Dollinger MM and Christ B: The generation of hepatocytes from mesenchymal stem cells and engraftment into murine liver. Nat Protoc 5: 617-627, 2010 .

3. Nombela-Arrieta C, Ritz J and Silberstein LE: The elusive nature and function of mesenchymal stem cells. Nat Rev Mol Cell Biol 12: 126-131, 2011

4. Schwartz RE, Reyes M, Koodie L, Jiang Y, Blackstad M, Lund T, Lenvik T, Johnson S, Hu WS and Verfaillie CM: Multipotent adult progenitor cells from bone marrow differentiate into functional hepatocyte-like cells. J Clin Invest 109: 1291-1302, 2002.

5. Lange C, Bassler P, Lioznov MV, Bruns H, Kluth D, Zander AR and Fiegel HC: Hepatocytic gene expression in cultured rat mesenchymal stem cells. Transplant Proc 37: 276-279, 2005.

6. Hong SH, Gang EJ, Jeong JA, Ahn C, Hwang SH, Yang IH, Park HK, Han $\mathrm{H}$ and Kim $\mathrm{H}$ : In vitro differentiation of human umbilical cord blood-derived mesenchymal stem cells into hepatocyte-like cells. Biochem Biophys Res Commun 330: 1153-1161, 2005.

7. Aurich I, Mueller LP, Aurich H, Luetzkendorf J, Tisljar K, Dollinger MM, Schormann W, Walldorf J, Hengstler JG, Fleig WE and Christ B: Functional integration of hepatocytes derived from human mesenchymal stem cells into mouse livers. Gut 56: 405-415, 2007.

8. Aurich H, Sgodda M, Kaltwasser P, Vetter M, Weise A, Liehr T, Brulport M, Hengstler JG, Dollinger MM, Fleig WE and Christ B: Hepatocyte differentiation of mesenchymal stem cells from human adipose tissue in vitro promotes hepatic integration in vivo. Gut 58 570-581, 2009

9. Jiang Y, Jahagirdar BN, Reinhardt RL, Schwartz RE, Keene CD, Ortiz-Gonzalez XR, Reyes M, Lenvik T, Lund T, Blackstad M, Du J, Aldrich S, Lisberg A, Low WC, Largaespada DA and Verfaillie CM: Pluripotency of mesenchymal stem cells derived from adult marrow. Nature 418: 41-49, 2002.

10. Di Nicola M, Carlo-Stella C, Magni M, Milanesi M, Longoni PD, Matteucci P, Grisanti S and Gianni AM: Human bone marrow stromal cells suppress T-lymphocyte proliferation induced by cellular or nonspecific mitogenic stimuli. Blood 99: 3838-3843, 2002.

11. Le Blanc K, Tammik C, Rosendahl K,Zetterberg E and Ringden O: HLA expression and immunologic properties of differentiated and undifferentiated mesenchymal stem cells. Exp Hematol 31: $890-896,2003$

12. Corcione A, Benvenuto F, Ferretti E, Giunti D, Cappiello V, Cazzanti F, Risso M, Gualandi F, Mancardi GL, Pistoia V and Uccelli A: Human mesenchymal stem cells modulate B-cell functions. Blood 107: 367-372, 2006.

13. Rasmusson I, Le Blanc K, Sundberg B and Ringden O: Mesenchymal stem cells stimulate antibody secretion in human B cells. Scand J Immunol 65: 336-343, 2007.

14. Jiang XX, Zhang Y, Liu B, Zhang SX, Wu Y, Yu XD and Mao N: Human mesenchymal stem cells inhibit differentiation and function of monocyte-derived dendritic cells. Blood 105: 4120-4126, 2005.

15. Ramasamy R, Fazekasova H, Lam EW, Soeiro I, Lombardi G and Dazzi F: Mesenchymal stem cells inhibit dendritic cell differentiation and function by preventing entry into the cell cycle. Transplantation 83: 71-76, 2007.
16. Sotiropoulou PA, Perez SA, Gritzapis AD, Baxevanis $C N$ and Papamichail M: Interactions between human mesenchymal stem cells and natural killer cells. Stem Cells 24: 74-85, 2006.

17. Peng L, Xie DY, Lin BL, Liu J, Zhu HP, Xie C, Zheng YB and Gao ZL: Autologous bone marrow mesenchymal stem cell transplantation in liver failure patients caused by hepatitis B: Short-term and long-term outcomes. Hepatology doi: 10.1002/hep.24434 [Epub ahead of print], 2011.

18. Le Blanc K, Rasmusson I, Sundberg B, Gotherstrom C, Hassan M, Uzunel $\mathrm{M}$ and Ringden O: Treatment of severe acute graft-versushost disease with third party haploidentical mesenchymal stem cells. Lancet 363: 1439-1441, 2004.

19. Ringden O, Uzunel M, Rasmusson I, Remberger M, Sundberg B, Lonnies H, Marschall HU, Dlugosz A, Szakos A, Hassan Z, Omazic B, Aschan J, Barkholt L and Le Blanc K: Mesenchymal stem cells for treatment of therapy-resistant graft-versus-host disease. Transplantation 81: 1390-1397, 2006.

20. Le Blanc K, Frassoni F, Ball L, Locatelli F, Roelofs H, Lewis I, Lanino E, Sundberg B, Bernardo ME, Remberger M, Dini G, Egeler RM, Bacigalupo A, Fibbe W and Ringden O: Mesenchymal stem cells for treatment of steroid-resistant, severe, acute graftversus-host disease: a phase II study. Lancet 371: 1579-1586, 2008.

21. Parekkadan B, van Poll D, Megeed Z, Kobayashi N, Tilles AW, Berthiaume $F$ and Yarmush ML: Immunomodulation of activated hepatic stellate cells by mesenchymal stem cells. Biochem Biophys Res Commun 363: 247-252, 2007.

22. van Poll D, Parekkadan B, Cho CH, Berthiaume F, Nahmias Y, Tilles AW and Yarmush ML: Mesenchymal stem cell-derived molecules directly modulate hepatocellular death and regeneration in vitro and in vivo. Hepatology 47: 1634-1643, 2008.

23. Liechty KW, MacKenzie TC, Shaaban AF, Radu A, Moseley AM, Deans R, Marshak DR and Flake AW: Human mesenchymal stem cells engraft and demonstrate site-specific differentiation after in utero transplantation in sheep. Nat Med 6: 1282-1286, 2000.

24. Le Blanc K: Immunomodulatory effects of fetal and adult mesenchymal stem cells. Cytotherapy 5: 485-489, 2003.

25. Khan M, Mohsin S, Khan SN and Riazuddin S: Repair of senescent myocardium by mesenchymal stem cells is dependent on the age of donor mice. J Cell Mol Med 15: 1515-1527, 2011.

26. Wilson A, Shehadeh LA, Yu H and Webster KA: Age-related molecular genetic changes of murine bone marrow mesenchymal stem cells. BMC Genomics 11: 229, 2010.

27. Erickson IE, van Veen SC, Sengupta S, Kestle SR and Mauck RL: Cartilage matrix formation by bovine mesenchymal stem cells in three-dimensional culture is age-dependent. Clin Orthop Relat Res 469: 2744-2753, 2011.

28. Katsara O, Mahaira LG, Iliopoulou EG, Moustaki A, Antsaklis A, Loutradis D, Stefanidis K, Baxevanis CN, Papamichail M and Perez SA: Effects of donor age, gender, and in vitro cellular aging on the phenotypic, functional, and molecular characteristics of mouse bone marrow-derived mesenchymal stem cells. Stem Cells Dev 20: 1549-1561, 2011.

29. Laschober GT, Brunauer R, Jamnig A, Singh S, Hafen U, Fehrer C, Kloss F, Gassner R and Lepperdinger G: Age-specific changes of mesenchymal stem cells are paralleled by upregulation of CD106 expression as a response to aninflammatory environment. Rejuvenation Res 14: 119-131, 2011.

30. Guguen-Guillouzo C, Campion JP, Brissot P, Glaise D, Launois B, Bourel M and Guillouzo A: High yield preparation of isolated human adult hepatocytes by enzymatic perfusion of the liver. Cell Biol Int Rep 6: 625-628, 1982.

31. Hengstler JG, Utesch D, Steinberg P, Platt KL, Diener B, Ringel M, Swales N, Fischer T, Biefang K, Gerl M, Bottger T and Oesch F: Cryopreserved primary hepatocytes as a constantly available in vitro model for the evaluation of human and animal drug metabolism and enzyme induction. Drug Metab Rev 32: 81-118, 2000.

32. Williams GM and Gunn JM: Long-term cell culture of adult rat liver epithelial cells. Exp Cell Res 89: 139-142, 1974.

33. Kallifatidis G, Rausch V, Baumann B, Apel A, Beckermann BM, Groth A, Mattern J, Li Z, Kolb A, Moldenhauer G, Altevogt P, Wirth T, Werner J, Schemmer P, Buchler MW, Salnikov AV and Herr I: Sulforaphane targets pancreatic tumour-initiating cells by NF-kappaB-induced antiapoptotic signalling. Gut 58: 949-963, 2009.

34. Zawada RJ, Kwan P, Olszewski KL, Llinas M and Huang SG: Quantitative determination of urea concentrations in cell culture medium. Biochem Cell Biol 87: 541-544, 2009. 
35. Zhang C, Kolb A, Buchler P, Cato AC, Mattern J, Rittgen W, Edler L, Debatin KM, Buchler MW, Friess H and Herr I: Corticosteroid co-treatment induces resistance to chemotherapy in surgical resections, xenografts and established cell lines of pancreatic cancer. BMC Cancer 6: 61, 2006

36. Wagner W, Feldmann RE Jr, Seckinger A, Maurer MH, Wein F, Blake J, Krause U, Kalenka A, Burgers HF, Saffrich R, Wuchter P, Kuschinsky W and Ho AD: The heterogeneity of human mesenchymal stem cell preparations-evidence from simultaneous analysis of proteomes and transcriptomes. Exp Hematol 34: 536-548, 2006

37. Pittenger MF and Marshak DR: Mesenchymal stem cells of human adult bone marrow. In: Stem Cell Biology. Marshak DR Gardner RL and Gottlieb D (eds). Cold Spring Harbor (NY): Cold Spring Harbor Laboratory Press, pp349-373, 2001.

38. Runge D, Runge DM, Jager D, Lubecki KA, Beer Stolz D, Karathanasis S, Kietzmann T, Strom SC, Jungermann K, Fleig WE and Michalopoulos GK: Serum-free, long-term cultures of human hepatocytes: maintenance of cell morphology, transcription factors, and liver-specific functions. Biochem Biophys Res Commun 269: 46-53, 2000.

39. Maitra B, Szekely E, Gjini K, Laughlin MJ, Dennis J, Haynesworth SE and Koc ON: Human mesenchymal stem cells support unrelated donor hematopoietic stem cells and suppress T-cell activation. Bone Marrow Transplant 33: 597-604, 2004.

40. Aurich H, Koenig S, Schneider C, Walldorf J, Krause P, Fleig WE and Christ B: Functional characterization of serum-free cultured rat hepatocytes for downstream transplantation applications. Cell Transplant 14: 497-506, 2005.

41. Koenig S, Aurich H, Schneider C, Krause P, Haftendorn R, Becker $\mathrm{H}$ and Christ B: Zonal expression of hepatocytic marker enzymes during liver repopulation. Histochem Cell Biol 128: 105-114, 2007.

42. Bartholomew A, Sturgeon C, Siatskas M, Ferrer K, McIntosh K, Patil S, Hardy W, Devine S, Ucker D, Deans R, Moseley A and Hoffman R: Mesenchymal stem cells suppress lymphocyte proliferation in vitro and prolong skin graft survival in vivo. Exp Hematol 30: $42-48,2002$.
43. Poncelet AJ, Denis D and Gianello P: Cellular xenotransplantation. Curr Opin Organ Transplant 14: 168-174, 2009.

44. Liu J, Lu XF, Wan L, Li YP, Li SF, Zeng LY, Zeng YZ, Cheng LH, $\mathrm{Lu}$ YR and Cheng JQ: Suppression of human peripheral blood lymphocyte proliferation by immortalized mesenchymal stem cells derived from bone marrow of Banna Minipig inbred-line. Transplant Proc 36: 3272-3275, 2004.

45. Saito T, Kuang JQ, Bittira B, Al-Khaldi A and Chiu RC: Xenotransplant cardiac chimera: immune tolerance of adult stem cells. Ann Thorac Surg 74: 19-24, 2002.

46. Grinnemo KH, Mansson A, Dellgren G, Klingberg D, Wardell E, Drvota V, Tammik C, Holgersson J, Ringden O, Sylven C and Le Blanc K: Xenoreactivity and engraftment of human mesenchymal stem cells transplanted into infarcted rat myocardium. J Thorac Cardiovasc Surg 127: 1293-1300, 2004.

47. Artrip JH, Kwiatkowski P, Michler RE, Wang SF, Tugulea S, Ankersmit J, Chisholm L, McKenzie IF, Sandrin MS and Itescu S: Target cell susceptibility to lysis by human natural killer cells is augmented by alpha(1,3)-galactosyltransferase and reduced by alpha(1, 2)-fucosyltransferase. J Biol Chem 274: 10717-10722, 1999.

48. Inverardi L, Clissi B, Stolzer AL, Bender JR, Sandrin MS and Pardi R: Human natural killer lymphocytes directly recognize evolutionarily conserved oligosaccharide ligands expressed by xenogeneic tissues. Transplantation 63: 1318-1330, 1997.

49. Gojo S, Harper D, Down J,Awwad M and Cooper DK: Differential expression of Galalpha1, 3Gal epitopes on fetal and adult porcine hematopoietic cells. Xenotransplantation 9: 297-300, 2002.

50. Harrower TP, Richards A, Cruz G, Copeman L, Dunnett SB and Barker RA: Alpha Gal is widely expressed in embryonic porcine stem cells and neural tissue. Neuroreport 13: 481-485, 2002. 